\title{
Antena MMIC Espiral
}

\section{Leonardo Augusto de Santana, Emerson Ferreira Vilhena e José Felipe Almeida}

Resumo-Trata-se de uma geometria em espiral de formato retangular a qual é baseada na análise de um monopolo espiral. É conhecido que a configuração de monopolo espiral permite uma larga faixa de operação em torno da freqüência central. $O$ modelo proposto é construído impresso sobre um substrato, na forma de uma antena de microfita. Verifica-se que para este tipo de microfita é observado um considerável acréscimo de largura de banda.

Palavras-Chave-Antena monopolo espiral, microfita, largura de banda.

Abstract-This work deal with a geometry in spiral rectangular format which is based on the analysis of a spiral monopole. There is known that this configuration allows a bandwidth of operation around the central resonant frequency. The considered model is printed on a substrate, in the form of a microstrip antenna. So, is verified that for this type of microstrip a considerable addition in the bandwidth is observed.

Index Terms - Monopole spiral antenna, bandwidth, microstrip antenna.

\section{INTRODUÇÃO}

Uma das dificuldades das antenas de microfita convencionais é a faixa estreita de operação [1-3]. Dessa forma várias técnicas têm sido empregada para resolver tal problema [4]. Esta classe de antena se constituem em circuitos integrados que utilizam estruturas planares (MMICs (monolithic microwave integrated circuits)).

Em contraposição ao problema das microfitas estão as antenas monopolo espirais. Estas antenas possuem diagrama de radiação e impedância de entrada sem muita variação dentro de um largo espectro e por isso são conhecidas como independentes da freqüência [5-6]. Em [7], por exemplo, os autores utilizaram uma técnica numérica para simular aquilo que chamaram de monopolo espiral horizontal e da mesma maneira em [8], sendo que de forma vertical [9], em relação ao plano de terra, com o objetivo de analisar alguns parâmetros dessa antenas.

A finalidade deste trabalho é a de apresentar uma antena espiral com a configuração de MMIC. A qual também pode ser chamada de antena de microfita espiral retangular. Toda a geometria da proposta desse trabalho é fundamentada, portanto, nas antenas de microfitas [1-3] e nas idéias de análise feitas para monopolos espiral [7-8]. Dessa forma, um modelo de uma antena bastante simples será confeccionado e seu diagrama de perdas será apresentado a partir de medição experimental.

L. A. Santana E. F. Vilhena e J. F. Almeida, Instituto de Estudos Superiores da Amazônia- IESAM, Belém do Pará, Brasil, E-mail: leonardo.a.santana@gmail.com, felipe@lane.ufpa.br

\section{Modelo e Resultados Obtidos}

O modelo da antena apresentada como projeto deste trabalho é do tipo microfita. Esta antena é alimentada por uma linha de fita, a qual deverá compor toda a antena em quebras ortogonais regulares, ou seja, na forma de uma espira. Na Figura a seguir é mostrada a geometria de toda a estrutura.

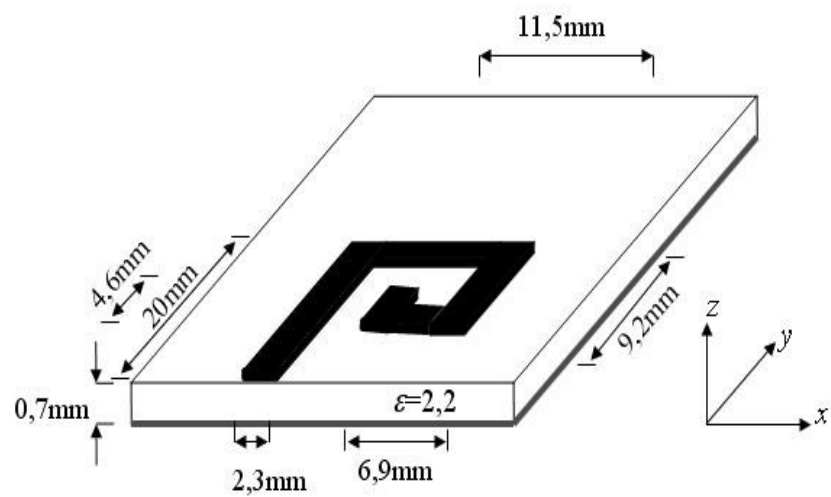

Fig. 1. Ilustração geométrica da antena proposta.

Para a confecção do modelo da antena, da Fig.1, foi utilizada uma placa de circuito elétrico, dupla face, especificada pela placa Duroid 5880. A permissividade elétrica relativa desse material vale $\varepsilon_{r}=2,2$. Esse material não é de fácil aquisição e foi gentilmente cedido pelo LEA/PPGEE/UFPA.

$O$ circuito a ser impresso parte do dimensionamento de uma linha de fita. Dessa forma, a largura da linha metálica foi estabelecida em $W=2,3 \mathrm{~mm}$. A espessura do material dielétrico (Duroid 5880) vale $h=0,7 \mathrm{~mm}$. A impedância característica dessa linha de transmissão é de $50 \Omega$.

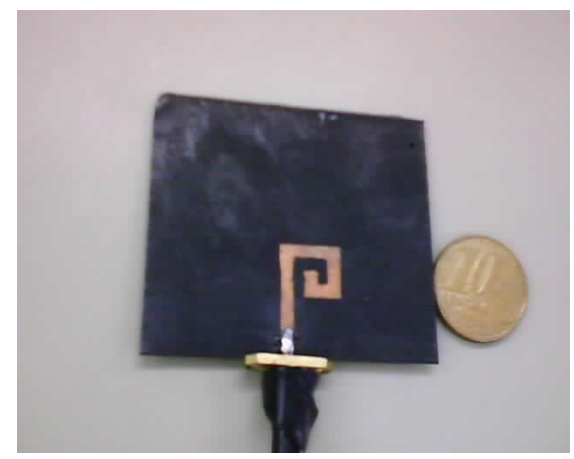

Fig. 2. Antena MMIC espiral. 


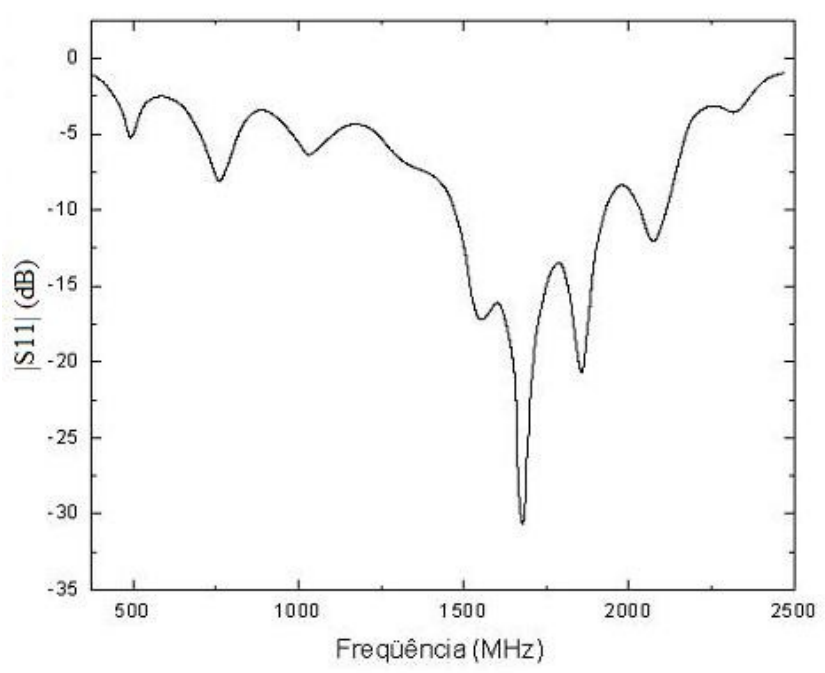

Fig. 3. Gráfico de medidas da antena da Fig.2.

As Figs. 2 e 3 apresentam a antena confeccionada neste projeto e o gráfico das suas medidas experimentais, respectivamente. A Fig.2 apresenta uma comparação das dimensões da antena com o diâmetro de uma moeda conhecida. Na Fig. 3, note-se que para a freqüência central de $1,683 \mathrm{GHz}$ a perda de retorno (coeficiente relexão) da antena é medido em -30 dB. Nesta freqüência, a antena opera dentro da faixa compreendida entre $1,5-2,0 \mathrm{GHz}$. Isto representa uma largura de banda en torno de 30\%. Estes teste foram feitos no analisador Site Master, gentilmente cedido pela Empresa Amazônia Celular.

\section{ConclusõES}

O resultado final deste trabalho é uma antena do tipo microfita espiral ou antena MMIC espiral. Com a geometria apresentada, obteve-se uma antena com um bom desempenho na faixa compreendida entre $1,5 \mathrm{GHz}$ e $2,0 \mathrm{GHz}$. Esta faixa representa uma largura de banda de aproximadamente $30 \%$, em torno da freqüência central de 1,68 GHz. A base desse projeto são as microfitas e as antenas monopolo de forma espiral, as quais mesmo otimizadas atingem a mesma ordem. Entretanto, o principal aspecto relacionado com a microfita proposta é o valor apresentado, de largura de banda, em comparação com as outras geometria de microfitas convencionais. Como é o caso das de forma retangular e circular. Além disso, quando comparada com um monopolo espiral, as antenas impressas, devido sua portabilidade e estrutura compacta, são bem mais adequadas aos sistemas da tecnologia em vigor. A contribuição desse trabalho reside no fato de se ter apresentado uma configuração geométrica para as microfitas a qual permite operação com largura de banda sem o uso de técnicas especiais. Entre as propostas de trabalhos futuros, está a análise teórica deste tipo de antena, por exemplo, considerando-se uma análise na teoria de fractais. Não foram apresentados resultados comparativos com o monopolo, citado no trabalho, devido ao caráter de confecção daquela antena, isto é, não se tem um modelo real analisado experimentalmente.

Nos trabalhos aqui citados, os resultados que permitiram uma averiguação foram considerados apenas como uma estimativa de valores feitos por simulação (no caso a antena monopolo), os quais levam em conta parâmetros normalizados em vários comprimento de onda. Fica, portanto, difícil apresentar comparações, pois não apresentam resultados experimentais. Todavia, o modelo aqui proposto é um aperfeiçoamento das idéias destes trabalhos e o qual, devido a pouca aplicabilidade da faixa de operação escolhida, sugere um projeto para este tipo de antena. Ou seja esta antena contribui para um trabalho de valor excepcionalmente de cunho didático e que pode ser usado como ponto de partida para a construção de uma dessas antenas capaz de operar na faixa desejada.

\section{REFERÊNCIAS}

[1] J. F. Almeida, Análise Fotônica em Estrutura de Microfita Planar Usando o Método FDTD com Processamento Paralelo, UFPa: tese de doutorado, 2004.

[2] R. Garg, P. Bhartia, I. Bahl, and A. Ittipiboon, Microstrip Antenna Design Handbook. Norwood: Artech, 2001.

[3] E. Chang, S. A. Long, and W. F. Richards, "Experimental Investigation of Electrically Thick Rectangular Microstrip Antennas," IEEE Trans. Antennas Propagat., vol. 43, pp. 767-772, 1986.

[4] J. F. Almeida, C. L. S. S. Sobrinho, and R. O. Santos, "Analysis by FDTD Method of a Microstrip Antenna with PBG Considering the Substrate Thickness Variation," Journal of Microwave and Optoelectronics, vol. 3, no.3, pp.41-48, Dec. 2003.

[5] C. A. Balanis, Antenna Theory: Analysis and Design, 2nd ed., New York: John Wiley, 1997.

[6] W. L. Curtis, "Spiral antennas", IEEE Trans. on Antennas and Propagat., pp. 298-306, May 1960.

[7] J. A. Kaiser, "The archimedean two-wire spiral antenna", IEEE Trans. on Antennas and Propagat., pp. 312-323, May 1960.

[8] K. Q. da Costa, V. A. Dmitriev e C. L. da S. S. Sobrinho, "Análise e Otimização de um Monopólo Espiral Horizontal,” 2º Momag, Campinas/SP, 2005.

[9] K. Q. da Costa, V. Dmitriev, C. Rodrigues, "Fractal Spiral Monopoles: theoretical analysis and bandwidth optimization," SBMO/IEEE, MTTS, IMOC, Brasília-DF, Brasil, July 2005. 